\title{
CONVECTION STUDY BY PIV METHOD WITHIN HORIZONTAL LIQUID LAYER EVAPORATING INTO INERT GAS FLOW
}

\author{
Aleksei Kreta ${ }^{1,2, *}$, and Yuriy Lyulin ${ }^{2,3}$ \\ ${ }^{1}$ Novosibirsk State University, Novosibirsk, Russia \\ ${ }^{2}$ Kutateladze Institute of Thermophysics SB RAS, Novosibirsk, Russia \\ ${ }^{3}$ Tomsk Polytechnic University, Tomsk, Russia
}

\begin{abstract}
The paper is devoted to the experimental study of convection in a horizontal evaporating liquid layer (ethanol) of limited size under the action of gas flow (air). The two-dimensional velocity field in the liquid layer is obtained using the PIV method. The existence of a vortex convective flow within a liquid layer directed towards the gas flow has been revealed.
\end{abstract}

\section{Introduction}

Studying heat and mass transfer through the interface between liquid and gas is one of the most important problems nowadays. In the energy devices, the considered processes are implemented in mini- and microchannels of the apparatus such as heat pipes, film evaporators, two-phase cooling systems, etc. The intensive evaporating from the liquid layer surface under the gas flow causes various convective flows in the liquid layer. The shape and structure of the convection is quite complicated and can have a significant impact on the evaporation rate and hence the efficiency of heat exchangers. The convection flows within the evaporating liquid layer into inert gas, are actively studied experimentally [1-4] and theoretically [4-7] in the present time. Nevertheless works in this area is extremely insufficient for comprehensive understanding of the physics of processes related to heat and mass transfer through the interface between liquid and gas, as well as convection in the liquid layer.

The aim of this work is to experimentally study the structure of convective flows and to measure two-dimensional velocity field distribution in the liquid layer under the action of the gas flow using PIV method.

\section{Experimental setup}

The experimental studies were conducted on the setup shown in fig. 1 . The setup consists of the following main components: test section, gas and liquid loops, data acquisition system,

* Corresponding author: alexy.slav@yandex.ru 
thermal stabilization system, and optical techniques. Pure gas flows from the compressor to the test section. Gas mass flow rate at the inlet of the channel in the test section is set by means of mass flow controller. The liquid is injected into the test section by a precision syringe pump. The working liquid evaporates under the gas flow and the vapor-gas mixture flows to the outlet of the gas channel in test section. The vapor-gas mixture flow at the outlet is measured by a mass flow meter. The liquid temperature in the test section is determined by the temperature of the substrate base, which controlled with the help of thermoelectric module. The difference between the temperature of the gas and liquid does not exceed $0.1{ }^{0} \mathrm{C}$. The position of the interface level is controlled with an accuracy of 10 micrometers using a Schlieren technique and the syringe pump [1]. Recording of the convection flows in the liquid layer was carried out by the PIV technique.

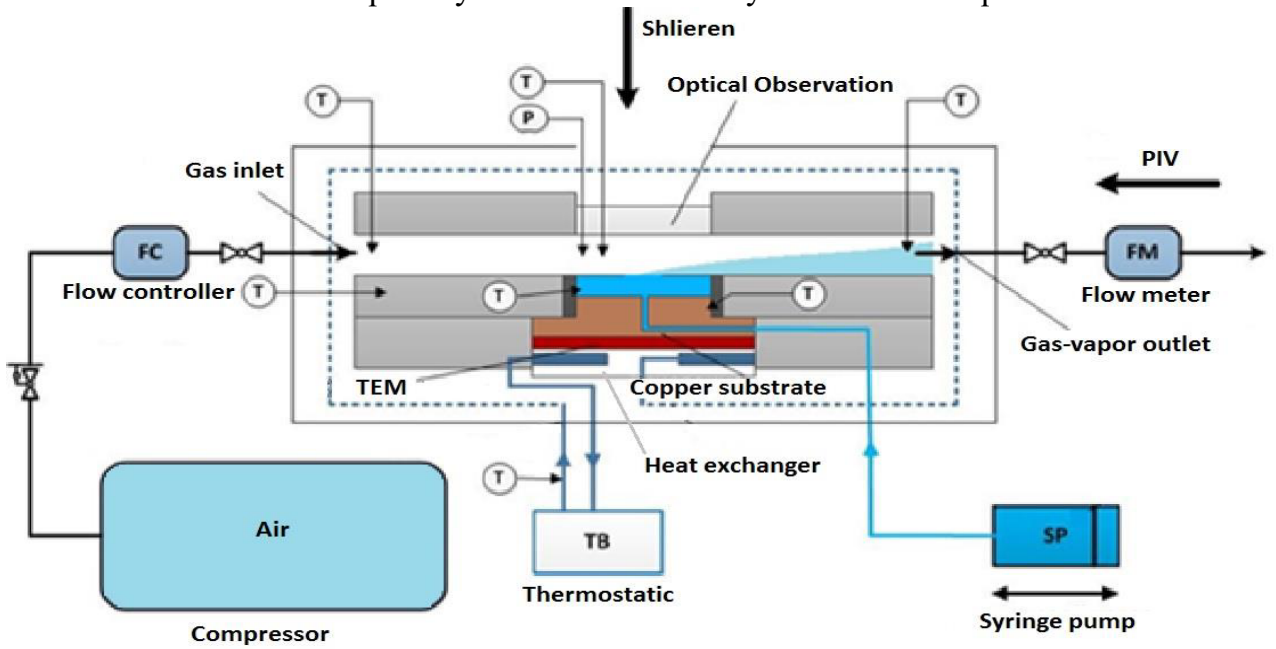

Fig. 1. Schematic diagram of the experimental setup.

The test section consists of several interconnected blocks. These blocks form a rectangular gas channel with a height of $3 \mathrm{~mm}$ and a width of $40 \mathrm{~mm}$, as well as a rectangular $40 \times 40 \mathrm{~mm} 2$ liquid chamber. A stainless steel plate with a square cut-out at the center is installed between the gas channel and the chamber with a liquid. The walls of the liquid chamber are made of transparent Plexiglas that gives the possibility to use PIV technique. The height of the liquid in the chamber varies from 1 to $10 \mathrm{~mm}$ and can be adjusted by means of a liquid substrate and special plates regulating the height. The cover of the test section is provided with transparent optical window installed above the liquid chamber.

\subsection{PIV measurements}

Recording of the convective structures in the liquid layer under action of the gas flow is carried out using the Particle Image Velocimetry (PIV) method. The schematic diagram of the method is shown in fig. 2. The method allows to determine the shape, velocity of the vortex structures in the liquid layer depending on various system parameters such as gas flow velocity, liquid temperature, the height of the liquid layer, etc. Detailed description of PIV method is given in [8]. 


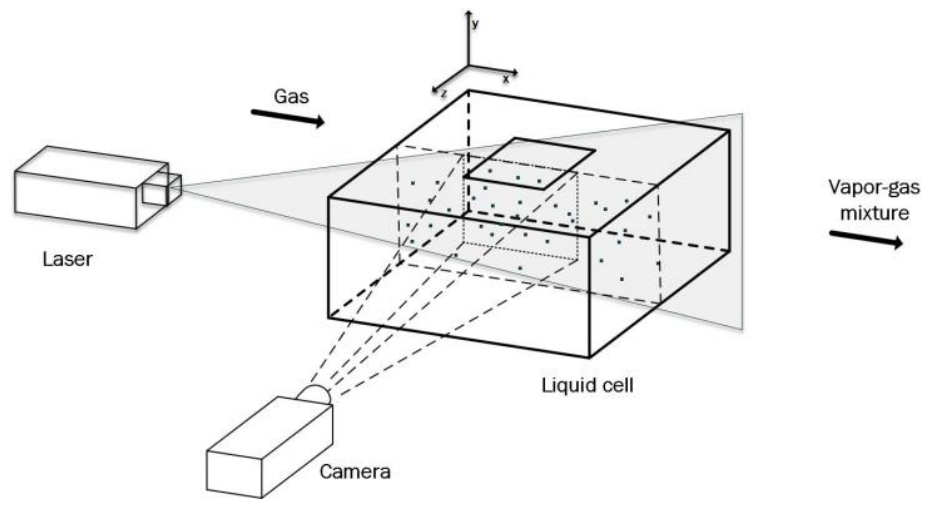

Fig. 2. Schematic diagram of the PIV system.

Instantaneous two-component velocity field is measured in the central cross section of the liquid layer directed towards the gas flow. In the beginning of the experiment, the particles (tracers) with a size of 5 microns are added to the liquid. The size and density of the tracers are chosen in such a way as to avoid the influence of the particles on the liquid flow pattern. A pulsed laser creates a thin $(300 \mu \mathrm{m})$ light knife and illuminates small particles. The particles positions at the time of two consecutive laser flashes are recorded on two frames of a digital camera. A digital camera is located completely perpendicular to the light sheet. The flow velocity is determined by calculating a displacement of the particles during the time between the laser flashes. The determination of the particles displacement is based on application of correlation methods to the images of a tracer particle using a regular decomposition into elementary regions. The data processing is carried out using the "ActualFlow" software program. The program implements two main methods for image processing: the standard and the iterative cross-correlation methods [9]. The standard crosscorrelation method is based on the employment of fast Fourier transform using the correlation theorem. However, for a more accurate calculation of the velocity vector is used an iterative cross-correlation method for calculation of two-component velocity field. The basic idea of the method consists in multiple processing of one and the same area using the result of processing at the previous iteration to estimate the processing parameters for the next iteration. As a consequence of this approach, it is possible to obtain a significant increase in the accuracy of the obtained data [9]. The cross-correlation method is used as the primary method to calculate two-component velocity field.

\section{Results}

The experiments were conducted at atmospheric pressure in a test section, and a stationary $3 \mathrm{~mm}$ thick liquid layer. Ethanol was used as the working liquid. The evaporation surface area was $100 \mathrm{~mm}^{2}$ with the corresponding $10 \times 10 \mathrm{~mm}$ cut-out in the plate. The gas flow rate ranged from 100 to $1000 \mathrm{ml} / \mathrm{min}$ with increment of $100 \mathrm{ml} / \mathrm{min}$ that corresponds to gas average velocity from $0.0139 \mathrm{~m} / \mathrm{s}(\mathrm{Re}=2.8)$ to $0.138 \mathrm{~m} / \mathrm{s}(\mathrm{Re}=28)$, respectively. The temperature of the "liquid-gas" system was maintained constant at a level of $20^{\circ} \mathrm{C}$ with a deviation less than $0.10 \mathrm{C}$.

Fig. 3 represents a two-component velocity field for a minimal $(100 \mathrm{ml} / \mathrm{min})$ and maximal $(1000 \mathrm{ml} / \mathrm{min})$ gas flow rates. The calculation of the average velocity of convective structures is conducted as follows. First, the area with a liquid vortex is determined visually. Next, a rectangular area with this vortex is selected in the "ActualFlow" program. The program displays a table with the coordinates and velocity projections along the axes $\mathrm{x}$ and $\mathrm{y}$ for each particle in the selected area. Particles with 
extremely low velocity vectors are removed from the table. Removed vectors are located near borders of the measuring area and outside of the liquid convective vortex. The velocity of each particle is determined by the formula:

$$
\mathrm{V}=\sqrt{ }\left(\left(\mathrm{V}_{\mathrm{x}}\right)^{2}+\left(\mathrm{V}_{\mathrm{y}}\right)^{2}\right)
$$

where, $V_{x}$ and $V_{y}$ - are $x$ and $y$ components of the velocity vector. The velocity of the convective vortex is calculated as the average value of the particle velocities in the measuring area.

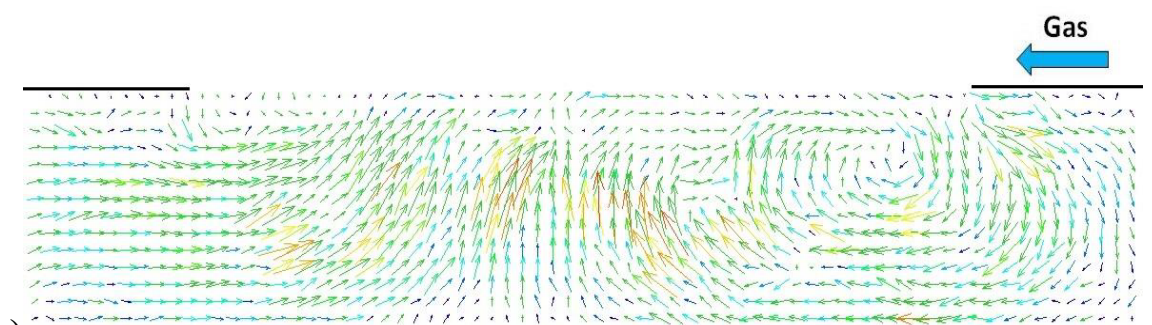

a)

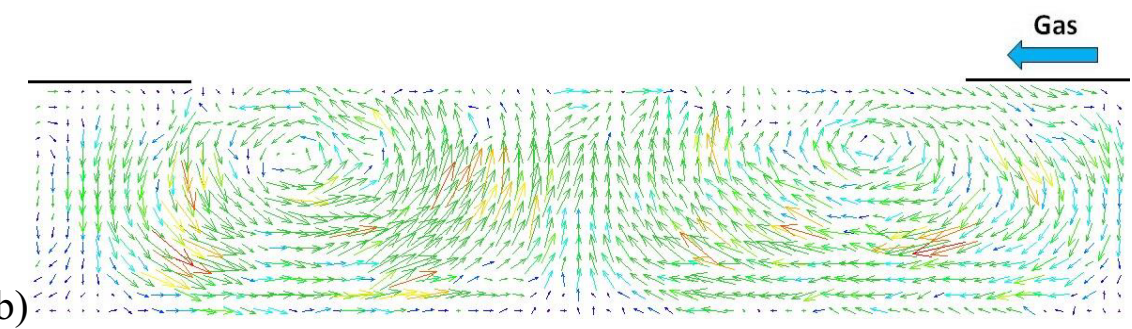

Fig. 3. Two-component velocity field. Liquid - ethanol. The layer thickness is $3 \mathrm{~mm}$; a) gas flow rate is $100 \mathrm{ml} / \mathrm{min}(\mathrm{Vgas}=0.0139 \mathrm{~m} / \mathrm{s}, \mathrm{Re}=2,8), \mathrm{b})$ gas flow rate is $1000 \mathrm{ml} / \mathrm{min}(\mathrm{Vgas}=0.138 \mathrm{~m} / \mathrm{s}, \mathrm{Re}$ $=28)$.

Fig. 3 (a) demonstrates the convective vortex at the initial section of the contact between liquid and gas. The direction of the vortex motion is opposite to the direction of the gas flow. The average velocity of the vortex is $0.18 \mathrm{~m} / \mathrm{s}$. Direction of a liquid flow opposite to the gas flow is due to the dominant influence of thermocapillary forces caused by the intensive evaporation at the interface. The gas flow cools the liquid surface causing a temperature gradient in the liquid layer that forces liquid to move against the gas flow. Fig. 3 (b) presents a two-component velocity field at a gas flow rate of $1000 \mathrm{ml} / \mathrm{min}$. It is found the formation of two symmetrical and inverse convective vortexes within the liquid layer at the maximum gas flow rate. The first vortex at the initial area of the gas-liquid contact is moving in counter-current direction of the gas flow. Governing factor of the convective vortex is the thermocapillary effect as in the previous case (the gas flow rate of 100 $\mathrm{ml} / \mathrm{min}$ ). The second vortex moves in the same direction as the gas flow. This liquid movement occurs due to the action of the gas shear stress and the effect of the first vortex structure. The velocities of the first and second vortex structures are equal to 0.2412 and $0.2416 \mathrm{~m} / \mathrm{s}$, respectively.

\section{Conclusion}

Convection study by PIV method in a horizontal liquid layer evaporating into the gas flow has been performed. Effect of gas flow on the convective flows in the liquid layer has been considered. It is shown that the increase of the gas flow velocity induces the number of 
convective vortex in horizontal evaporating liquid layer and growth of its velocities. For minimum gas flow rate $(100 \mathrm{ml} / \mathrm{min})$, only one convective structure moving against the gas flow was observed. When increasing the gas flow rate to $1000 \mathrm{ml} / \mathrm{min}$, another convective structure appears and moves along with the gas flow. Governing factor of the convective vortex moving in counter-current direction of the gas flow is the thermocapillary effect due to the intensive evaporation and the temperature decreasing near the attack border of the liquid.

\section{Acknowledgments}

The work was financially supported by the Russian Ministry of Education and Science (Project identifier RFMEFI61314X0011).

\section{References}

1. O.A. Kabov. Int. J. of Em. Multidisc. Liq. Sc. 2 (2010)

2. Yu.V. Lyulin, D.V. Feoktistov, I.A. Afanasiev, E.S. Chachilo, O.A. Kabov, G.V. Kuznetsov, Let. Tech. Phys. 41 (2015)

3. Yu.V. Lyulin, O.A. Kabov, Int. J. Heat Mass Transf. 70 (2014)

4. B. Scheid, J. Margerit, C.S. Iorio, L. Joannes, M. Heraud, P. Queeckers, P.C. Dauby, P. Colinet, Exp. Liq. 52 (2012)

5. O.N. Goncharova, O.A. Kabov, Int. J. Heat Mass Transf. 53 (2010)

6. O.N. Goncharova, O.A. Kabov, Int. J. Heat Mass Transf. 53 (2010)

7. H. Machrafi, C. S. Iorio, P.C. Dauby, Int. Phen. Heat Transf. 2 (2014)

8. H. Machrafi, C.S. Iorio, P.C. Dauby, Int. Phen. Heat Transf. 2 (2014)

9. C. Brossard, J. Monnier, P. Barricau, Opt. Diagn. Fl. (2009) 